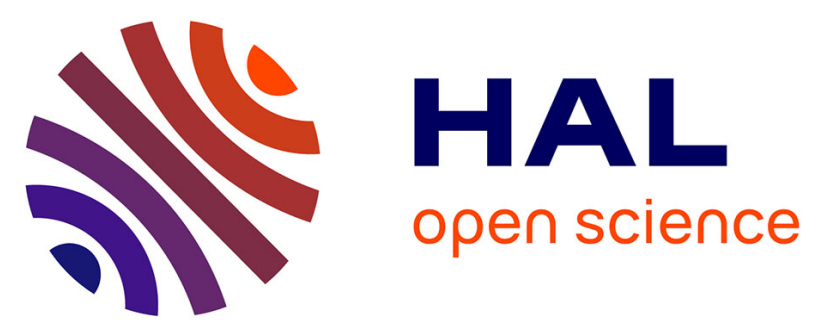

\title{
Osimertinib (Tagrisso®) : activité, indication et modalités d'utilisation dans les cancers bronchiques non à petites cellules
}

Etienne Giroux Leprieur, Alexis B Cortot, Jacques Cadranel, Marie Wislez

\section{- To cite this version:}

Etienne Giroux Leprieur, Alexis B Cortot, Jacques Cadranel, Marie Wislez. Osimertinib (Tagrisso®) : activité, indication et modalités d'utilisation dans les cancers bronchiques non à petites cellules. Bulletin du Cancer, 2016, 103 (10), pp.815-821. 10.1016/j.bulcan.2016.06.007 . hal-01371930

\section{HAL Id: hal-01371930 \\ https: / hal.sorbonne-universite.fr/hal-01371930}

Submitted on 26 Sep 2016

HAL is a multi-disciplinary open access archive for the deposit and dissemination of scientific research documents, whether they are published or not. The documents may come from teaching and research institutions in France or abroad, or from public or private research centers.
L'archive ouverte pluridisciplinaire HAL, est destinée au dépôt et à la diffusion de documents scientifiques de niveau recherche, publiés ou non, émanant des établissements d'enseignement et de recherche français ou étrangers, des laboratoires publics ou privés. 
Osimertinib (Tagrisso ${ }^{\circledR}$ ) : activité, indication et modalités d'utilisation dans les cancers bronchiques non à petites cellules

Osimertinib (Tagrisso®) : activity, indication and modality of use in non-small cell lung cancer

Etienne Giroux Leprieur ${ }^{1,2}$, Alexis B. Cortot ${ }^{3,4}$, Jacques Cadranel $^{5,6}$, Marie Wislez $^{5,6}$

1- Service de Pneumologie et Oncologie thoracique, APHP - Hôpital Ambroise Paré, 9 avenue Charles de Gaulle, F-92100 Boulogne-Billancourt

2- Laboratoire EA4340, Biomarqueurs en Cancérologie et Onco-Hématologie, UVSQ, Université Paris-Saclay, 9 avenue Charles de Gaulle, F-92100 Boulogne-Billancourt

3- Service de Pneumologie et Oncologie Thoracique, Hôpital Calmette, CHU Lille, F59000, Lille

4- UMR8161, Institut de Biologie de Lille, Lille

5- Service de Pneumologie, APHP - Hôpital Tenon, 4 rue de la Chine, F-75020 Paris

6- Sorbonne Universités, UPMC Univ Paris 06, GRC n04, Theranoscan, F-75252, Paris

Auteur correspondant : Marie Wislez, Service de Pneumologie, APHP - Hôpital Tenon, 4 rue de la Chine, F-75020 Paris ; marie.wislez@aphp.fr. 


\section{Résumé}

L'acquisition d'une mutation EGFR de résistance au niveau de l'exon 20 (T790M) est responsable de plus de la moitié des cas de résistance secondaire aux inhibiteurs de tyrosine kinase (ITK) de l'EGFR, prescrits en $1^{\text {ière }}$ ligne dans les cancers bronchiques non à petites cellules (CBNPC) de stade localement avancé ou métastatique, mutés EGFR. L'osimertinib (AZD9291, Tagrisso ${ }^{\circledR}$ ) est un inhibiteur irréversible d'EGFR de $3^{\text {ième }}$ génération, actif en cas de mutations activatrices et mutation T790M. Un essai de phase I avec extension de cohorte a montré son efficacité après échec des ITK de I'EGFR de $1^{\text {ière }}$ génération (erlotinib, gefitinib), avec des taux de réponse de $51 \%$, et jusqu'à $61 \%$ en cas de mutation T790M. La survie sans progression en cas de mutation T790M était de 9,6 mois. Le profil de toxicité est acceptable, avec essentiellement une toxicité digestive (diarrhées) et cutanée (rash). Des données préliminaires d'un essai de phase II ont confirmé ces résultats d'efficacité et de tolérance. La prescription d'osimertinib est conditionnée à la mise en évidence d'une mutation T790M dont la recherche au moment de la progression sous ITK peut être faite sur l'ADN tumoral circulant dans le plasma, avec des performances diagnostiques intéressantes ce qui en fait une alternative à la rebiopsie.

Mots-clés : cancer bronchique non à petites cellules ; EGFR ; T790M ; osimertinib ; AZD9291 ; ADN tumoral circulant 


\section{Abstract}

The acquisition of a resistance EGFR mutation in exon 20 (T790M) occurs in the half of the case of secondary resistance to EGFR tyrosine kinase inhibitors (TKI), given in first-line treatment in advanced EGFR-mutated non-small cell lung cancers (NSCLC). Osimertinib (AZD9291, Tagrisso ${ }^{\circledR}$ ) is a thirdgeneration, irreversible EGFR TKI, active in case of T790M mutation. A large phase I trial showed the efficacy of osimertinib after failure of first-generation EGFR TKI (erlotinib, gefitinib), with response rate at $51 \%$ and up to $61 \%$ in case of T790M mutation. Progression-free survival was 9.6 months in case of T790M. Toxicity profile was acceptable, with mainly digestive (diarrhea) and skin (rash) sideeffects. Preliminary data from a phase II trial confirmed these efficacy and safety data. Screening of T790M mutation at the time of progression with TKI can be performed in circulating tumor DNA in plasma, with good diagnostic performances.

Key-words: non-small cell lung cancer; EGFR; T790M; osimertinib; AZD9291; circulating tumor DNA 


\section{Introduction}

Le cancer bronchique non à petites cellules (CBNPC) est le type de cancer bronchique le plus fréquent. Dans la grande majorité des cas il est diagnostiqué à un stade avancé (localement avancé ou métastatique). Dans les pays européens, une mutation du gène de l'EGFR (Epidermal Growth Factor Receptor) est mise en évidence dans environ 10 à 15\% des CBNPC de stade avancé [1]. Dans plus de $80 \%$ des cas, il s'agit de mutations situées au niveau de l'exon 19 (délétion) ou de l'exon 21 (notamment la mutation L858R), qui induisent une activation constitutionnelle du domaine tyrosine kinase de l'EGFR et une sensibilité particulière aux inhibiteurs de tyrosine kinase ciblant EGFR (ITK EGFR). Les ITK EGFR dits de $1^{\text {ière }}$ génération sont le gefitinib et l'erlotinib. Ils ont montré leur efficacité

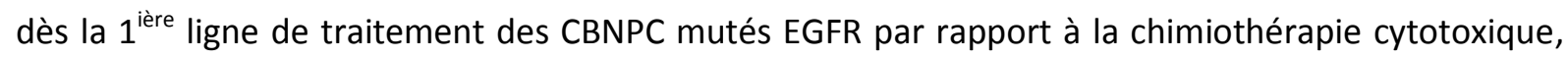
avec des survies sans progression (SSP, médiane) de 9 à 13 mois (ITK EGFR), versus 5 à 6 mois (chimiothérapie)] [2-5]. Cependant, une progression tumorale survient dans l'ensemble des cas avec une médiane de 9 à 10 mois (résistance secondaire). Dans la moitié des cas, le mécanisme moléculaire associé à cette progression est l'acquisition d'une mutation de résistance au niveau de l'exon 20 (mutation T790M) [6]. D'autres mécanismes comme une amplification de MET, une activation d'HER2, la transformation histologique en carcinome à petites cellules ou la transition épithélio-mésenchymateuse ont été décrits. L'afatinib est un ITK dit irréversible de l'EGFR, de $2^{\text {ième }}$ génération, initialement développé pour cibler la mutation T790M [7]. Cependant, les études cliniques n'ont pas pas montré d'efficacité de cette molécule aux doses recommandées, après progression sous ITK de $1^{\text {ière }}$ génération $[8,9]$; par ailleurs, il a été mis en évidence de manière similaire aux autres ITK l'apparition d'une mutation T790M comme mécanisme de résistance secondaire à l'afatinib [9]. L'afatinib a également reçu en 2013 l'Autorisation de Mise sur le Marché (AMM) dans les CBNPC de stade avancé avec mutation EGFR et naïfs de tout traitement $[10,11]$. L'osimertinib (AZD9291, Tagrisso $^{\circledR}$ ) est un ITK EGFR de $3^{\text {ième }}$ génération, développé pour cibler les CBNPC avec mutations EGFR activatrices et T790M. Après une Autorisation Temporaire d'Utilisation (ATU) dans le traitement des CBNPC avec mutation T790M après échec d'un ITK EGFR, ce 
médicament vient d'obtenir l'AMM en mars 2016 dans le traitement des CBNPC localement avancés ou métastatiques, avec mutation EGFR T790M, sans restriction sur la ligne de traitement. Cette revue décrit les principales données d'efficacité de ce produit et ses modalités d'utilisation.

\section{Données d'efficacité préclinique}

L'AZD9291 a montré in vitro une efficacité sur les lignées cellulaires de CBNPC avec mutations EGFR, dont la mutation T790M [12]. Ainsi, en terme d'inhibition de prolifération tumorale, l'IC50 de I'AZD9291 était de $11 \mathrm{nM} / \mathrm{L}$ et de 40nM/L pour les lignées T790M+ H1975 et PC-9VanR, respectivement, versus $>400 \mathrm{nM} / \mathrm{L}$ avec l'afatinib et $>4000 \mathrm{nM} / \mathrm{L}$ avec les ITK de $1^{\text {ière }}$ génération (erlotinib et gefitinib). De plus, l'AZD9291 était également actif sur la lignée PC-9 (avec délétion exon 19) avec une IC50 de 8nM/L, et très peu actif sur les lignées EGFR sauvages Calu-3 et H2073, avec des IC50>400nM/L (versus <100nM/L avec l'afatinib). L'efficacité de l'AZD9291 a été confirmée sur des modèles murins de CBNPC mutés EGFR (T790M- et T790M+) que ce soit sur xénogreffes sur souris nude ou sur des modèles oncogéniques murins de CBNPC mutés EGFR [12].

\section{Données d'efficacité clinique}

\section{Phase I}

Les résultats de l'essai de phase I ont été publiés l'année dernière [13]. Trente-et-un patients avec CBNPC muté EGFR (ou ayant eu un bénéfice clinique sous ITK EGFR selon les critères de Jackman [14]) et ayant progressé sous ITK EGFR ont été inclus dans la phase d'escalade de doses $(20 \mathrm{mg} / \mathrm{j}$ à 240mg/j). II n’a pu être mis en évidence de dose limitante en termes de toxicité. Secondairement, 222 patients, dont 138 avec mutation T790M, ont été randomisés entre 5 cohortes d'expansion (20mg, 40mg, 80mg, 160mg, 240mg). Dans cette phase d'expansion, $61 \%$ des patients étaient de 
sexe féminin, avec un âge médian de 60 ans, $60 \%$ d'ethnie asiatique, 96\% avec un adénocarcinome, avec en médiane 2 lignes d'ITK EGFR préalables. La moitié avait une mutation de l'exon 19, 29\% une mutation L858R, 5\% une autre mutation activatrice d'EGFR, et $16 \%$ aucune mutation ou non recherchée. Le taux de réponse objective (TRO) était de $51 \%$, et le taux de contrôle de $84 \%$, quelle que soit la dose. Parmi les patients avec mutation T790M évaluables pour la réponse ( $n=127)$, le TRO était de $61 \%$, tandis qu'il n'était que de $21 \%$ pour les patients sans mutation T790M ( $n=61)$. La survie sans progression (SSP) était de 9,6 mois pour les patients avec mutation T790M, versus 2,8 mois pour les patients sans mutation T790M. Des données actualisées ont été communiquées récemment sur les patients ayant reçu la dose retenu pour l'AMM de $80 \mathrm{mg} / \mathrm{j}$ et avec mutation T790M ( $n=63$ ) [15]. Pour ces patients, le TRO actualisé était de $71 \%$ et le taux de contrôle de $93 \%$. La durée médiane de réponse était de 9,6 mois (sur 43 patients évaluables). La SSP actualisée était de 9,7 mois. Concernant le sous-groupe de patients ayant reçu l'osimertinib en $1^{\text {ière }}$ ligne (à la dose de $80 \mathrm{mg}$ ou de $160 \mathrm{mg} / \mathrm{j})(\mathrm{n}=60)$, le TRO était de $77 \%$, le taux de contrôle de la maladie de $98 \%$, la durée médiane de réponse non atteinte (avec 53\% des patients encore en réponse à 18 mois), la SSP de 19,3 mois [16]. Cinq patients avaient une mutation T790M de novo et tous ont eu une réponse partielle sous osimertinib, avec une durée de réponse entre 12,2 et 20,7 mois.

\section{Phase II}

L'essai AURA2 est un essai de phase II qui a testé l'osimertinib $(80 \mathrm{mg} / \mathrm{j})$ chez des patients avec CBNPC muté EGFR et une mutation T790M objectivée par rebiopsie au moment de la progression sous ITK EGFR ( $n=210)$. Des résultats préliminaires combinant les résultats de cet essai de phase II et des 201 patients de la phase d'extension de l'essai de phase I ayant une mutation T790M et ayant reçu la dose de $80 \mathrm{mg} / \mathrm{j}$ ont été présentés cette année à l'European Lung Cancer Conference (ELCC) (soit 411 patients au total) [15]. Sur 397 patients évaluables au moment de l'analyse, le TRO était de 
$66 \%$, et le taux de contrôle de $91 \%$. La durée médiane de réponse était de 12,5 mois (sur 262 patients évaluables). La SSP était de 11 mois $(n=411)$.

\section{Phases III et place de l'osimertinib en $1^{\text {ière }}$ ligne de traitement}

Un essai de phase III (NCT02151981) est actuellement en cours de recrutement, comparant l'osimertinib $(80 \mathrm{mg} / \mathrm{j})$ à un doublet sel de platine + pemetrexed dans les CBNPC de stade IV mutés EGFR ayant progressé sous ITK EGFR et porteur d'une mutation T790M. L'objectif principal est la SSP et un cross-over est possible pour l'osimertinib après la chimiothérapie.

Un autre essai de phase III FLAURA (NCT02296125) compare en double aveugle en $1^{\text {ière }}$ ligne de traitement l'osimertinib $(80 \mathrm{mg} / \mathrm{j})$ à un ITK de $1^{\text {ière }}$ génération (gefitinib ou erlotinib) dans les CBNPC de stade IV mutés EGFR. L'objectif principal est la SSP, et un cross-over pour l'osimertinib est possible après échec de l'ITK de $1^{\text {ière }}$ génération dans le bras contrôle. Cet essai est actuellement ouvert, et pose la question du moment d'introduction de ces molécules de $3^{\text {ième }}$ génération dans la stratégie thérapeutique des CBNPC mutés EGFR, l'idée étant d'empêcher l'apparition de la T790M lors de l'introduction dès la $1^{\text {ière }}$ ligne, et ainsi de retarder l'apparition d'une résistance due à d'autres mécanismes. L'AMM de l'osimertinib est le traitement des CBNPC localement avancés ou métastatiques, avec mutation EGFR T790M, sans restriction sur la ligne de traitement. La mutation T790M n'est retrouvée que très rarement au moment du diagnostic avant traitement par ITK EGFR. Le libellé de l'AMM correspond donc dans la majorité des cas à une situation de progression sous ITK de $1^{\text {ière }}$ ou $2^{\text {ième }}$ génération. Cependant, le développement de techniques très sensibles de séquençage de l'ADN permettant de mettre en évidence la T790M dès le diagnostic dans près de $70 \%$ des cas dans certaines séries [17], pose la question de l'opportunité de donner l'osimertinib dans ces situations dès la $1^{\text {ière }}$ ligne, alors même que les résultats de l'essai FLAURA ne sont pas connus. 


\section{Toxicité}

Les effets secondaires rapportés avec l'osimertinib sont des effets de classe, communs avec les autres ITK EGFR, mais leur fréquence semble bien moindre qu'avec les ITK EGFR de $1^{\text {ière }}$ et $2^{\text {ième }}$ génération, probablement du fait d'une activité moindre sur les cellules EGFR sauvage [10]. Dans l'essai de phase I ( $n=253$ au total), l'incidence des effets secondaires de grade 3-5 était de 32\%, dont $13 \%$ imputables au médicament, avec $7 \%$ de diminution de la posologie et $6 \%$ d'arrêt dû à un effet secondaire [13]. Les effets secondaires les plus fréquents étaient la diarrhée (47\%), le rash acnéiforme (40\%), les nausées (22\%), la baisse d'appétit (21\%), la sécheresse cutanée $(20 \%)$ et le prurit (19\%). Les effets secondaires de grade 3-5 les plus fréquents étaient les diarrhées (2\%), l'anémie (2\%), la dyspnée (2\%). Sur les 7 décès toxiques rapportés, un seul a été relié au traitement (pneumonie). Il y a eu 6 cas de pneumopathies interstitielles (2\%) Tous ont eu une évolution satisfaisante de leur atteinte pulmonaire suite à l'arrêt de l'osimertinib. Les données préliminaires de la phase II poolée n'ont pas montré de différence en termes de profil de toxicité [15]. Des effets secondaires de grade 1-2 unguéaux (paronychie, onycholyse...) et ophtalmologiques (vision floue, kératite, blépharite...) ont aussi été décrits.

\section{Pharmacocinétique}

Les analyses pharmacocinétiques ont montré que l'exposition, la concentration plasmatique maximale et l'aire sous la courbe temps - concentration plasmatique, augmentaient proportionnellement à la posologie d'osimertinib $[13,18]$. La demi-vie est de $48 \mathrm{~h}$. L'état d'équilibre est obtenu après 15 jours de traitement. II n’y a pas de différence de pharmacocinétique entre les patients asiatiques et non-asiatiques. La prise alimentaire n'influence pas la biodisponibilité du produit de manière significative. Ces résultats sont compatibles avec une prise orale unique 
quotidienne, à dose unique, indépendamment des horaires de repas [18]. L'osimertinib est métabolisé principalement par le CYP3A4 et le CYP3A5, et est un inhibiteur compétitif des transporteurs BCRP, ce qui a des conséquences en termes d'interactions médicamenteuses (cf rubrique « En pratique » ci-dessous).

\section{Détection de la mutation T790M}

Dans les essais de phase I et II, la mutation T790M devait être mise en évidence à partir d'une nouvelle biopsie réalisée au moment de la progression sous ITK EGFR. Cependant, il n'est pas toujours possible d'effectuer une nouvelle biopsie au moment de la progression sous ITK EGFR (pas de re-biopsie possible dans environ $20 \%$ des cas sur des données de cohorte prospective nationale [19]). De plus, il semblerait que la présence d'une mutation T790M varierait entre les différents sites tumoraux. Ainsi, Hata et al. ont montré, à partir de 30 patients avec mutation EGFR qui ont eu des biopsies sur plusieurs sites tumoraux au moment de la progression sous ITK EGFR, une disparité entre la positivité des recherche de la T790M sur les différents sites [20]. Sur 22 patients qui avaient eu une rebiopsie à la fois sur le site thoracique et sur le système nerveux central (SNC), une mutation T790M était retrouvée sur le site thoracique pour 12 patients. Sur ces 12 patients, il n'était pas retrouvé la mutation T790M sur le prélèvement du SNC chez 10 patients. Pour les 10 patients sans mutation T790M au niveau thoracique, aucune mutation T790M n'était mise en évidence au niveau du SNC. De plus, une hétérogénéité de la présence de la mutation T790M au sein de la biopsie thoracique a été mise en évidence chez 3 patients [20].

Comme pour la détection des mutations sensibilisatrices d'EGFR [21-24], l'utilisation de l'ADN tumoral circulant pour la détection de la mutation T790M est intéressante: utilisation facile, possiblement répétée, et reflétant l'hétérogénéité tumorale. Plusieurs travaux ont montré la faisabilité et la robustesse de cette approche $[22,25,26]$. Des premiers résultats sur les données de l'essai de phase I ont été publiés en 2015 [27]. Pour la détection des mutations sensibilisatrices 
d'EGFR la sensibilité est entre 82 et $87 \%$ (en fonction des techniques utilisées) et la spécificité de $97 \%$, et pour la T790M la sensibilité est de $73-81 \%$ et la spécificité entre $58 \%$ à $67 \%$ [27]. Cette spécificité moins bonne reflète peut être l'hétérogénéité tumorale des clones T790M au niveau des tissus, prélèvement de référence. Des données actualisées ont été présentées à l'ELCC cette année sur 216 patients de la phase I avec possibilité de comparaison biopsie et plasma [28]. La méthode utilisée sur le plasma était la PCR digitale (BEAMing digital PCR (dPCR)). La sensibilité de détection de la mutation T790M sur le plasma était de $70 \%$ (versus $82-86 \%$ pour les mutations EGFR sensibilisatrices). De manière intéressante, il était possible de " rattraper » les faux-négatifs dans le plasma ( $n=18$ ) en utilisant une technique alternative (ddPCR ou cobas) dans 14 cas sur 18 . Le taux de « faux-positifs » pour T790M dans le plasma (i.e. avec T790M négative sur la biopsie) était de 31\% (versus 3-4\% pour les mutations sensibilisatrices), possiblement du fait de l'hétérogénéité tumorale de la mutation T790M. Le taux de réponse à l'AZD9291 en cas de présence d'une mutation T790M dans le plasma était équivalent à celui retrouvé sur la biopsie : $63 \%$ et $62 \%$, respectivement. Par contre, le taux de réponse était de $46 \%$ en cas d'absence de T790M sur le plasma, versus $26 \%$ si absence sur biopsie. De manière équivalente, la SSP était de 9,7 mois (médiane) avec l'AZD9291, que la mutation T790M ait été retrouvée sur le plasma ou sur la biopsie. Dans les plasmas T790M négatifs, l'analyse de la biopsie permet de « rattraper » les faux-négatifs du plasma (SSP de 16,5 mois avec l'AZD9291 si tumeur T790M positive, versus 2,8 mois si tumeur également T790M négative, $\mathrm{p}<0,001)$. Ces résultats incitent donc fortement à rechercher la mutation T790M au moment de la progression sous ITK EGFR sur le plasma. La présence d'une mutation T790M sur I'ADN tumoral circulant autorise la prescription de l'osimertinib, dont l'AMM est conditionnée par la mise en évidence de la mutation T790M que ce soit sur la biopsie ou dans le plasma. En cas d'absence de mutation T790M sur le plasma, la recherche doit être poursuivie sur une nouvelle biopsie. L'European Medicines Agency n'impose pas un test particulier pour la mise en évidence de la mutation T790M sur le plasma. 
De manière intéressante, tout comme le suivi des clones mutants EGFR (exons 19 et 21) dans le plasma sous ITK EGFR de $1^{\text {ière }}$ ou $2^{\text {ième }}$ génération, il est possible de suivre l'évolution du clone muté T790M pendant le traitement par AZD9291, avec diminution très rapide en quelques jours de la fraction mutée dans le plasma [29].

\section{Mécanismes de résistances à l'osimertinib}

Peu de données sont actuellement disponibles concernant les mécanismes de résistance à l'osimertinib. Quelques séries de cas avec biopsies effectuées à la progression sous osimertinib sont publiées. II s'agit toujours de patients ayant reçu l'osimertinib après progression sous ITK EGFR de $1^{\text {ère }}$ ou $2^{\text {nde }}$ génération. Dans ce cas, les clones résistants peuvent émerger soit des clones porteurs de la mutation sensibilisatrice initiale, soit des clones porteurs de la mutation sensibilisatrice et de la mutation T790M. De ce fait, une importante hétérogénéité des profils moléculaires à progression sous osimertinib est attendue. L'analyse de l'ADN tumoral circulant dans le plasma de 15 patients traités par osimertinib a montré au moment de la progression l'apparition d'une mutation EGFR de résistance C797S dans 6 cas (40\%) [30]. Chez 5 autres patients, il était noté la persistance de la mutation T790M sans acquisition de nouvelle mutation de résistance, et dans 4 cas la disparition de la mutation T790M avec persistance de la mutation EGFR sensibilisatrice initiale. Oxnard et al. ont présenté récemment les résultats de l'analyse de l'ADN tumoral circulant (par ddPCR) à la progression sous osimertinib chez 67 patients avec CBNPC présentant une mutation sensibilisatrice de l'EGFR et une mutation T790M lors de la progression sous AZD9291 [31]. Quinze patients (22\%) avaient acquis une mutation C797S, tous avec persistance d'une mutation T790M associée. La mutation C797S tendait à être plus fréquente en cas de mutation de l'exon 19 (13/43, 30\%) qu'en cas de mutation L858R $(2 / 24,8 \%, p=0,06)$. Trente-deux patients $(48 \%)$ n'avaient pas de mutation T790M détectable dans le plasma malgré la présence de la mutation sensibilisatrice. D’autres mécanismes de résistance à l'osimertinib indépendants de l'EGFR ont été décrits, comme une amplification de 
HER2 ou une amplification de MET [31,32]. Enfin, une série coréenne de 4 patients traités par osimertinib a montré au moment de la progression une transformation en cancer bronchique à petites cellules, une surexpression de MAPK1/AKT3, une surexpression de FGF2 et une délétion de PTEN [33]. Ces résultats préliminaires méritent d'être confirmés sur de plus larges effectifs. Les mécanismes de résistance sous osimertinib donné en $1^{\text {ère }}$ ligne ne sont pas encore connus.

\section{Traitement à la progression}

Des données préliminaires in vitro ont apporté des précisions sur la sensibilité aux traitements ultérieurs en cas d'acquisition d'une mutation d'EGFR C797S sous AZD9291 [34]. Si les mutations T790M et C797S sont situées en trans (i.e. sur 2 copies différentes d'ADN), les cellules seront sensibles à une combinaison d'ITK EGFR de $1^{\text {ière }}$ et de $3^{\text {ième }}$ génération. A l'inverse, si les mutations sont en cis (i.e. situées sur le même chromosome), les cellules seront résistantes aux ITK EGFR, en monothérapie ou en combinaison. De manière intéressante, si les cellules avec mutation activatrice d'EGFR acquièrent la mutation C797S sans mutation T790M, au décours d'un traitement par AZD9291 en $1^{\text {ière }}$ ligne, les cellules seront résistantes à l'AZD9291 mais resteront sensibles aux ITK EGFR de $1^{\text {ière }}$ génération.

Par ailleurs, l'impact thérapeutique d'une amplification d'HER2 ou de MET sous AZD9291 reste pour le moment inconnu, et nécessite des études spécifiques.

\section{Conclusion}

L'osimertinib est un nouvel inhibiteur d'EGFR avec une activité notable en cas de mutation de résistance T790M, et un très bon profil de tolérance. Son AMM dans les CBNPC avec mutation EGFR T790M n'apporte pas de restriction sur la ligne de traitement, ce qui ne correspond pas au libellé de I'ATU ni aux données validées par les essais qui concernent son utilisation en cas de progression 
après un ITK EGFR de $1^{\text {ière }}$ ou $2^{\text {ième }}$ génération. Les résultats de l'essai de phase III FLAURA permettront peut-être de préciser son intérêt dès la $1^{\text {ière }}$ ligne en termes de bénéfice de SSP et de survie globale. Enfin, la recherche de mutation T790M peut être faite sur plasma par analyse de I'ADN tumoral circulant. Des études sur les mécanismes de résistance à l'osimertinib sont en cours (en particulier acquisition d'une mutation EGFR C797S). D'autres ITK EGFR de $3^{\text {ième }}$ génération sont par ailleurs en développement (BI 1482694 (Boehringer Ingelheim) et EGF816 (Novartis)). 


\section{En pratique}

L'osimertinib est commercialisé par le laboratoire AstraZeneca sous le nom de Tagrisso ${ }^{\circledR}$, sous la forme de comprimés à $40 \mathrm{mg}$ et $80 \mathrm{mg}$. Son indication est le traitement des patients adultes atteints d'un cancer bronchique non à petites cellules (CBNPC) localement avancé ou métastatique, avec mutation EGFR T790M.

Posologie : $80 \mathrm{mg} / \mathrm{j}$ en prise unique, en dehors ou pendant un repas, à horaire fixe. En cas d'intolérance, une décroissance à $40 \mathrm{mg} / \mathrm{j}$ est possible.

\section{Bilan pré-thérapeutique ou surveillance}

- J1 : bilan biologique sanguin (NFS, ionogramme, créatinine, transaminases, bilirubine), ECG, test de grossesse le cas échéant

- Si indication pendant le traitement: ECG, NFS, ionogramme, créatinine, transaminases, bilirubine

\section{Instauration du traitement si à J1 :}

- $\quad$ ASAT/ALAT :

$0 \leq 2,5 \times$ normal en l'absence de métastases hépatiques,

$0 \leq 5 \times$ normal en présence de métastases hépatiques,

- Bilirubine totale $\leq 1,5 \times$ normal,

- QTc normal (<450 ms)

\section{Contre-indications}

- Hypersensibilité à la substance active ou à l'un des excipients

- Association au millepertuis

\section{Précautions d'emploi}

- Antécédents de pneumopathie interstitielle diffuse, de pneumopathie interstitielle diffuse médicamenteuse, de pneumopathie radique ayant nécessité un traitement par corticoïdes, ou tout signe de pneumopathie interstitielle diffuse cliniquement active

- Intervalle QTc > $470 \mathrm{~ms}$

\section{Effets indésirables possibles décrits}

- Toxicité digestive : diarrhées

- Toxicité cutanée : rash, stomatite, périnoxysis

- Toxicité cardiaque : allongement du QTc (arrêt définitif si symptomatique)

- Toxicité pulmonaires : pneumopathie interstitielle (arrêt définitif)

- Toxicité hématologique : leuco-neutropénie, thrombopénie

Interactions médicamenteuses : inducteurs puissants du CYP3A (contre-indication: phénytoïne, rifampicine, carbamazépine, millepertuis...), inducteurs modérés du CYP3A4 (précaution d'emploi : bosentan, efavirenz, étravirine, modafinil...), substrats de BCRP avec index thérapeutique étroit. Absence d'interaction avec les modificateurs du $\mathrm{pH}$ gastrique. 
Conflit d'intérêt : $A B C$ a participé à des réunions d'experts pour le laboratoire Astra-Zeneca Source de financement : aucune 


\section{Bibliographie}

1. Barlesi F, Mazieres J, Merlio J-P, Debieuvre D, Mosser J, et al. Routine molecular profiling of patients with advanced non-small-cell lung cancer: results of a 1-year nationwide programme of the French Cooperative Thoracic Intergroup (IFCT). Lancet 2016;387:1415-26.

2. Mitsudomi T, Morita S, Yatabe Y, Negoro S, Okamoto I, et al. Gefitinib versus cisplatin plus docetaxel in patients with non-small-cell lung cancer harbouring mutations of the epidermal growth factor receptor (WJTOG3405): an open label, randomised phase 3 trial. Lancet Oncol 2010;11:121-8.

3. Rosell R, Carcereny E, Gervais R, Vergnenegre A, Massuti B, et al. Erlotinib versus standard chemotherapy as first-line treatment for European patients with advanced EGFR mutationpositive non-small-cell lung cancer (EURTAC): a multicentre, open-label, randomised phase 3 trial. Lancet Oncol 2012;13:239-46.

4. Maemondo M, Inoue A, Kobayashi K, Sugawara S, Oizumi S, et al. Gefitinib or chemotherapy for non-small-cell lung cancer with mutated EGFR. N Engl J Med 2010;362:2380-8.

5. Zhou C, Wu YL, Chen G, Feng J, Liu XQ, et al. Erlotinib versus chemotherapy as first-line treatment for patients with advanced EGFR mutation-positive non-small-cell lung cancer (OPTIMAL, CTONG-0802): a multicentre, open-label, randomised, phase 3 study. Lancet Oncol 2011;12:735-42.

6. Kuiper JL, Heideman D a. M, Thunnissen E, Paul MA, van Wijk AW, et al. Incidence of T790M mutation in (sequential) rebiopsies in EGFR-mutated NSCLC-patients. Lung Cancer 2014;85:1924. 
7. Li D, Ambrogio L, Shimamura T, Kubo S, Takahashi M, et al. BIBW2992, an irreversible EGFR/HER2 inhibitor highly effective in preclinical lung cancer models. Oncogene 2008;27:4702-11.

8. Sos ML, Rode HB, Heynck S, Peifer M, Fischer F, et al. Chemogenomic profiling provides insights into the limited activity of irreversible EGFR Inhibitors in tumor cells expressing the T790M EGFR resistance mutation. Cancer Res 2010;70:868-74.

9. Kim Y, Ko J, Cui Z, Abolhoda A, Ahn JS, et al. The EGFR T790M mutation in acquired resistance to an irreversible second-generation EGFR inhibitor. Mol Cancer Ther 2012;11:784-91.

10. Sequist LV, Yang JC-H, Yamamoto N, O’Byrne K, Hirsh V, et al. Phase III study of afatinib or cisplatin plus pemetrexed in patients with metastatic lung adenocarcinoma with EGFR mutations. J Clin Oncol 2013;31:3327-34.

11. Wu Y-L, Zhou C, Hu C-P, Feng J, Lu S, et al. Afatinib versus cisplatin plus gemcitabine for first-line treatment of Asian patients with advanced non-small-cell lung cancer harbouring EGFR mutations (LUX-Lung 6): an open-label, randomised phase 3 trial. Lancet Oncol 2014;15:21322.

12. Cross DAE, Ashton SE, Ghiorghiu S, Eberlein C, Nebhan CA, et al. AZD9291, an Irreversible EGFR TKI, Overcomes T790M-Mediated Resistance to EGFR Inhibitors in Lung Cancer. Cancer Discov 2014;4:1046-61.

13. Jänne PA, Yang JC-H, Kim D-W, Planchard D, Ohe Y, et al. AZD9291 in EGFR inhibitor-resistant non-small-cell lung cancer. N Engl J Med 2015;372:1689-99.

14. Jackman D, Pao W, Riely GJ, Engelman JA, Kris MG, et al. Clinical definition of acquired resistance to epidermal growth factor receptor tyrosine kinase inhibitors in non-small-cell lung cancer. J Clin Oncol 2010;28:357-60. 
15. Yang JC-H, Ramalingam SS, Jänne PA, Cantarini M, Mitsudomi T. Osimertinib (AZD9291) in pretreated pts with T790M-positive advanced NSCLC: updated Phase 1 (P1) and pooled Phase 2 (P2) results. European Lung Cancer Conference 2016, Geneva (Switzerland): abstr LBA2_PR.

16. Ramalingam SS, Yang JC-H, Lee $\mathrm{C}$, Kurata $\mathrm{T}$, Kim D, et al. Osimertinib as first-line treatment for EGFR mutation-positive advanced NSCLC: updated efficacy and safety results from two Phase I expansion cohorts. European Lung Cancer Conference 2016, Geneva (Switzerland): abstr LBA1_PR.

17. Chen L-Y, Molina-Vila MA, Ruan S-Y, Su K-Y, Liao W-Y, et al. Coexistence of EGFR T790M mutation and common activating mutations in pretreatment non-small cell lung cancer: $\mathrm{A}$ systematic review and meta-analysis. Lung Cancer 2016;94:46-53.

18. Planchard D, Brown KH, Kim D-W, Kim S-W, Ohe Y, et al. Osimertinib Western and Asian clinical pharmacokinetics in patients and healthy volunteers: implications for formulation, dose, and dosing frequency in pivotal clinical studies. Cancer Chemother Pharmacol 2016;77:767-76.

19. Chouaid C, Dujon C, Do P, Monnet I, Madroszyk A, et al. Feasibility and clinical impact of rebiopsy in advanced non-small cell lung cancer: a prospective multicenter study in a real-world setting (GFPC study 12-01). Lung Cancer 2014;86:170-3.

20. Hata A, Katakami N, Yoshioka H, Kaji R, Masago K, et al. Spatiotemporal T790M Heterogeneity in Individual Patients with EGFR-Mutant Non-Small-Cell Lung Cancer after Acquired Resistance to EGFR-TKI. J Thorac Oncol 2015;10:1553-9.

21. Zhang $H$, Liu D, Li S, Zheng $Y$, Yang $X$, et al. Comparison of EGFR signaling pathway somatic DNA mutations derived from peripheral blood and corresponding tumor tissue of patients with advanced non-small-cell lung cancer using liquidchip technology. J Mol Diagn JMD $2013 ; 15: 819-26$. 
22. Sorensen BS, Wu L, Wei W, Tsai J, Weber B, et al. Monitoring of epidermal growth factor receptor tyrosine kinase inhibitor-sensitizing and resistance mutations in the plasma DNA of patients with advanced non-small cell lung cancer during treatment with erlotinib. Cancer 2014;120:3896-901.

23. Douillard J-Y, Ostoros G, Cobo M, Ciuleanu T, Cole R, et al. Gefitinib treatment in EGFR mutated caucasian NSCLC: circulating-free tumor DNA as a surrogate for determination of EGFR status. J Thorac Oncol 2014;9:1345-53.

24. Luo J, Shen L, Zheng D. Diagnostic value of circulating free DNA for the detection of EGFR mutation status in NSCLC: a systematic review and meta-analysis. Sci Rep 2014;4:6269.

25. Sakai K, Horiike A, Irwin DL, Kudo K, Fujita Y, et al. Detection of epidermal growth factor receptor T790M mutation in plasma DNA from patients refractory to epidermal growth factor receptor tyrosine kinase inhibitor. Cancer Sci 2013;104:1198-204.

26. Zheng $D$, Ye X, Zhang MZ, Sun Y, Wang JY, et al. Plasma EGFR T790M ctDNA status is associated with clinical outcome in advanced NSCLC patients with acquired EGFR-TKI resistance. Sci Rep 2016;6:20913.

27. Thress KS, Brant R, Carr TH, Dearden S, Jenkins S, et al. EGFR mutation detection in ctDNA from NSCLC patient plasma: A cross-platform comparison of leading technologies to support the clinical development of AZD9291. Lung Cancer 2015;90:509-15.

28. Oxnard GR, Thress KS, Alden R, Lawrance R, Paweletz $C P$, et al. Plasma genotyping for predicting benefit from osimertinib in patients (pts) with advanced NSCLC. European Lung Cancer Conference 2016, Geneva (Switzerland): abstr 1350_PR. 
29. Vallée A, Audigier-Valette C, Herbreteau G, Merrien J, Tessonnier L, et al. Rapid clearance of circulating tumor DNA during treatment with AZD9291 of a lung cancer patient presenting the resistance EGFR T790M mutation. Lung Cancer 2016;91:73-4.

30. Thress KS, Paweletz CP, Felip E, Cho BC, Stetson D, et al. Acquired EGFR C797S mutation mediates resistance to AZD9291 in non-small cell lung cancer harboring EGFR T790M. Nat Med 2015;21:560-2.

31. Oxnard GR, Thress KS, Paweletz CP, Stetson D, Dougherty B, et al. Mechanisms of Acquired Resistance to AZD9291 in EGFR T790M Positive Lung Cancer. J Thorac Oncol 2015;10s: abstr 17.06 .

32. Planchard D, Loriot $\mathrm{Y}$, André F, Gobert A, Auger N, et al. EGFR-independent mechanisms of acquired resistance to AZD9291 in EGFR T790M-positive NSCLC patients. Ann Oncol $2015 ; 26: 2073-8$.

33. Kim TM, Song A, Kim D-W, Kim S, Ahn Y-O, et al. Mechanisms of Acquired Resistance to AZD9291: A Mutation-Selective, Irreversible EGFR Inhibitor. J Thorac Oncol 2015;10:1736-44.

34. Niederst MJ, Hu H, Mulvey HE, Lockerman EL, Garcia AR, et al. The Allelic Context of the C797S Mutation Acquired upon Treatment with Third-Generation EGFR Inhibitors Impacts Sensitivity to Subsequent Treatment Strategies. Clin Cancer Res 2015;21:3924-33. 